D) Check for updates

Cite this: RSC Adv., 2021, 11, 4441

rsc.li/rsc-advances

\title{
Retraction: Salvianolic acid B inhibits inflammatory response and cell apoptosis via the PI3K/Akt signaling pathway in IL-1 $\beta$-induced osteoarthritis chondrocytes
}

\author{
Laura Fisher
}

Retraction of 'Salvianolic acid B inhibits inflammatory response and cell apoptosis via the PI3K/Akt signalling pathway in IL-1ß-induced osteoarthritis chondrocytes' by Bin Zhu et al., RSC Adv., 2018, 8, 36422-36429, DOI: $10.1039 /$ C8RA02418A.

The Royal Society of Chemistry hereby wholly retracts this RSC Advances article due to concerns with the reliability of the data. The images in the article were screened by an image integrity expert who confirmed that some of the western blots images in this paper had been duplicated in other articles. There are no common authors between the papers.

The Col II band in Fig. 3B of this paper has been duplicated as the p62 band in Fig. 4A of ref. 1.

One of the blots in the control band (GAPDH) in Fig. 3D has also been reused as a blot in Fig. 2C of ref. 1 and in Fig. 4A of ref. 2. The authors were asked to provide the raw data for this article but did not respond. Given the significance of the concerns about the validity of the data, and the lack of raw data, the findings presented in this paper are not reliable.

The authors have been informed but have not responded to any correspondence regarding the retraction.

Signed: Laura Fisher, Executive Editor, RSC Advances

Date: $7^{\text {th }}$ January 2021

\section{References}

1 Z. Tang, L. Yang and X. Zhang, RSC Adv., 2017, 7, 56406-56416.

2 H. Yang and S. Wu, RSC Adv., 2018, 8, 21816-21822. 